\title{
Review \\ Is subjective duration a signature of coding efficiency?
}

\author{
David M. Eagleman ${ }^{1,2, *}$ and Vani Pariyadath $^{1}$ \\ ${ }^{1}$ Department of Neuroscience, and ${ }^{2}$ Department of Psychiatry, Baylor College of Medicine, \\ Houston, TX 77030, USA
}

\begin{abstract}
Perceived duration is conventionally assumed to correspond with objective duration, but a growing literature suggests a more complex picture. For example, repeated stimuli appear briefer in duration than a novel stimulus of equal physical duration. We suggest that such duration illusions appear to parallel the neural phenomenon of repetition suppression, and we marshal evidence for a new hypothesis: the experience of duration is a signature of the amount of energy expended in representing a stimulus, i.e. the coding efficiency. This novel hypothesis offers a unified explanation for almost a dozen illusions in the literature in which subjective duration is modulated by properties of the stimulus such as size, brightness, motion and rate of flicker.
\end{abstract}

Keywords: time; duration; repetition suppression; illusion; oddball; proliferation effect

\section{INTRODUCTION}

The field of time perception has experienced a surge in research in the last decade (Buhusi \& Meck 2005; Eagleman et al. 2005; Ivry \& Schlerf 2008; Lin et al. 2008). While the psychophysical and neural data related to subjective duration have grown richer, there is no single accepted theory that explains how duration is encoded in the brain. Our purpose here is to review present models of duration perception, propose a new unified framework, explain how various findings in the literature are explained by that framework and outline new predictions.

The separation in time between two events-say, the onset of a light and its subsequent offset — can be judged by observers at many different time scales, from milliseconds to days (Mauk \& Buonomano 2004). We focus here on timing mechanisms that underlie judgements at the 'automatic' or 'direct sensation' time scales, i.e. on the scale of tens to hundreds of milliseconds (timing of longer scales, such as seconds and minutes and months are categorized as 'cognitive' and appear to be underpinned by entirely different neural mechanisms; Rammsayer 1999; Lewis \& Miall 2003; Buhusi \& Meck 2005). Although perceived duration is conventionally assumed to mirror objective duration, it is subject to illusory distortions of many sorts (Eagleman 2008). One example is the oddball effect: a novel or 'oddball' stimulus presented in a train of repeated stimuli appears to last longer in duration than the repeated stimuli (Tse et al. 2004; Pariyadath \& Eagleman 2007). Similarly, the first

* Author and address for correspondence: Department of Neuroscience, Baylor College of Medicine, Houston, TX 77030, USA (eagleman@bcm.edu).

One contribution of 14 to a Theme Issue 'The experience of time: neural mechanisms and the interplay of emotion, cognition and embodiment'. presentation of the repeated stimulus appears longer in duration than successive presentations (Rose \& Summers 1995). With illusions such as these in mind, we revisit recent models of timing.

A traditional model proposed to account for interval timing over short scales is a 'counter' model, in which internally generated pulses, or 'ticks', are collected up and integrated during the presence of a stimulus (Creelman 1962; Treisman 1963). This model is proposed to account for duration distortions in the following way: assume that the brain has access to the roughly constant rate of its own information processing, and that an increase in the rate of internal information processing (because of increased attention, fear, etc.) causes the counter to count more ticks. If the brain's assessment of duration is based on the integrated number of ticks, it would conclude that more objective time had passed between two events. Several authors have appealed to versions of this counter model to explain the duration distortion triggered by the oddball or the first stimulus (Rose \& Summers 1995; Ranganath \& Rainer 2003; Tse et al. 2004; Ulrich et al. 2006). In this framework, an increase in arousal caused by the appearance of an unexpected (oddball) stimulus leads to a transient increase in the tick rate of an internal clock. Thus, the accumulator collects a larger number of ticks in the same time period, and the oddball's duration is judged as longer.

However, the idea of a clock-like counter has found little support in physiology. An alternative model has proposed that the passage of time can be encoded in the evolving patterns of activity in neural networks (Buonomano \& Mauk 1994; Buonomano \& Merzenich 1995; Mauk \& Buonomano 2004; Karmarkar \& Buonomano 2007; Ivry \& Schlerf 2008). For example, imagine that every time a red cue light turns on, 
a specific spatio-temporal pattern of activity is triggered in the visual cortex. At $100 \mathrm{~ms}$ after the light turns on, a particular set of neurons may be active; shortly afterward, these neurons will activate other neurons, which will activate other neurons, and so on-leading to a specific pattern of neural activity that progresses into a different snapshot of active cells at every moment. When a salient event happens $100 \mathrm{~ms}$ after the cue light (say, the delivery of a juice reward), the snapshot of neurons that happened to be active at that moment is imprinted by the strengthening of their weights. In other words, the way the network evolves through time can code for time itself. In another version of the model, the ongoing neural activity of the network is not encoded in continuous spiking, but instead is carried in the state of intracellular signals, such as calcium concentrations (Buonomano \& Merzenich 1995); this expands the notion of the 'state' of the network from spikes to parameters which influences how spikes will be received and sent. A close cousin of this model relies on oscillating membrane potentials in individual neurons: if the phases of the oscillations are reset by a triggering event (the red light), then each successive moment in time can be encoded by the unique pattern of the relative phases of all the members of the population (Buhusi \& Meck 2005). Although these 'state-dependent' models are appealing, they await experimental validation and potentially suffer from the difficulty of making them work in noisy environments. In the context of this paper, the major challenge for the state-dependent model is to account for illusions of duration. After all, while it is difficult to generate a reproducible pattern of activity in a neural network, it is even more difficult to speed up or slow down the passage of the pattern through neural tissue without getting a new pattern entirely (but see Yamazaki \& Tanaka (2005) for an attempt in a noise-free artificial neural network).

\section{A NEW HYPOTHESIS FOR SUBJECTIVE DURATION: THE INFLUENCE OF REPETITION}

Having reviewed current models for duration encoding, we now propose a new hypothesis: the amount of neural energy required to represent a stimulus is proportional to, or at least influences, the subjective duration assigned to that stimulus (Pariyadath \& Eagleman 2007, 2008; Eagleman 2008). Note that our hypothesis is not necessarily incompatible with previous models, but, as will be shown below, may open the study of duration encoding to experiments easily performed with humans. The aim of this paper is to marshal the evidence supporting this framework.

\section{PREDICTABILITY INFLUENCES DURATION}

Duration dilations of unexpected stimuli (Rose \& Summers 1995; Hodinott-Hill et al. 2002; Ranganath \& Rainer 2003; Tse et al. 2004; Kanai \& Watanabe 2006; Ulrich et al. 2006; Pariyadath \& Eagleman 2007) have been called a subjective 'expansion of time' during the oddball (Tse et al. 2004). However, note that the psychophysical results could equally be interpreted as a duration contraction of the repeated stimuli, rather than an expansion of the first or oddball stimulus.

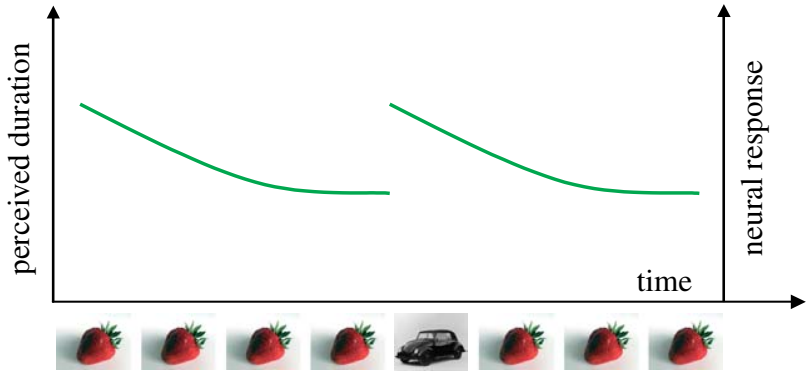

Figure 1. Cartoon of the main idea: the first stimulus in a repeated train, as well as an oddball stimulus, appears to last longer than the other stimuli. This pattern of diminishing duration perception with repetition appears to parallel the neural phenomenon of repetition suppression.

With this in mind, we have proposed that this pattern of duration distortions seems to parallel the pattern of neural activity seen with stimulus repetition (figure 1 ; Pariyadath \& Eagleman 2007).

Specifically, the amplitude of the neural response (firing rates) in higher cortical areas quickly diminishes after repeated presentations of a stimulus (Fahy et al. 1993; Li et al. 1993; Rainer \& Miller 2000), an effect generally known as repetition suppression (Henson \& Rugg 2003; Wark et al. 2007). In humans, repetition suppression has been observed in event-related potentials using electroencephalography (EEG) Grill-Spector et al. 2006) in the blood oxygen-level dependent (BOLD) response using functional magnetic resonance imaging (fMRI; Henson \& Rugg 2001) and also using positron emission tomography (Buckner et al. 1995) and magnetoencephalography (Noguchi et al. 2004; Ishai et al. 2006). Although it has been interpreted as stimulus-specific fatigue, more recent frameworks have interpreted repetition suppression as reflecting an increasing efficiency of representation (Desimone \& Duncan 1995; Wiggs \& Martin 1998; Grill-Spector et al. 2006). In that view, with repeated presentations of a stimulus, a sharpened representation or a more efficient encoding is achieved in the neural network coding for the object, affording lower metabolic costs. Our hypothesis states that this differential response to novel versus repeated stimuli maps on to perceived duration: a suppressed neural response corresponds to a shorter perceived duration.

The oddball studies traditionally use stimuli lasting for hundreds of milliseconds, but the principles apply for stimuli briefer than $100 \mathrm{~ms}$, i.e. stimuli that are too brief to make explicit temporal judgements. We were recently able to demonstrate this by developing a novel variation of the traditional flicker fusion frequency paradigm. In flicker fusion experiments, a light is rapidly turned on and off: at a low frequency, flicker is perceived, while at a high frequency, the light appears to be steady. The frequency at which the perception switches from flicker to a steady light is called the critical flicker fusion threshold (CFFT). Critically, CFFT experiments make use of a single stimulus that is presented repeatedly. Because there are subjective duration differences when viewing repeated versus novel stimuli, we hypothesized that the CFFT would change if the rapid stimulus could somehow be made novel each time it appeared. 
(a)

(i)

(ii)

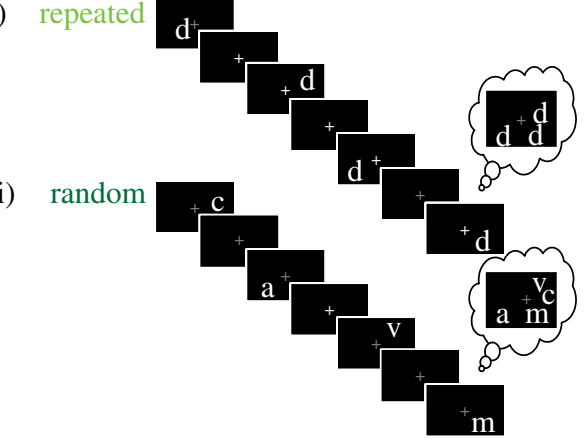

(b)

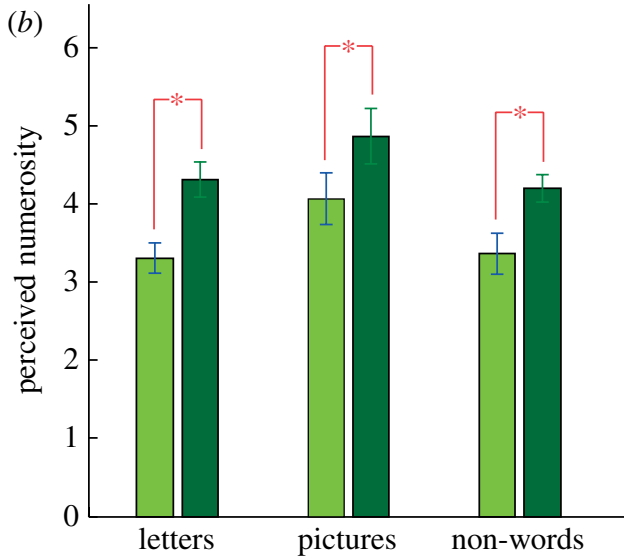

Figure 2. Repeated stimuli subjectively proliferate less than random stimuli. (a) Example sequences of stimulus presentation and perceived numerosity for repeated and random stimuli ('letters'). (b) Number of stimuli perceived to be present for various repeated (light green bars) and random (dark green bars) stimuli. Participants report more stimuli present on screen when the stimuli are different from when they are repeated $\left({ }^{*} p<0.05\right.$, paired $t$-tests). Error bars represent s.e.m.

To accomplish this, we presented stimuli serially at different locations on a computer screen. Although only one stimulus was present at any moment, more than one appeared to temporally overlap on screen due to visual persistence, a phenomenon that a briefly presented stimulus appears to last longer than the time it was physically presented (Efron 1970; Bowen et al. 1974; Di Lollo 1977). We refer to this perceived multiplicity of stimuli as the proliferation effect (Pariyadath \& Eagleman 2008). We employed two conditions: in the first, the same stimulus was presented ('repeated', figure $2 a$ ); in the second, different stimuli were presented ('random', figure $2 a$ ). Participants were required to report the number of stimuli subjectively present on screen at any one moment of time, i.e. how many characters appeared to share screen time.

Participants' estimates of how many characters they perceived on screen simultaneously varied significantly between the repeated and random conditions (figure $2 b$ ). At a $50 \mathrm{~Hz}$ presentation rate, for example, observers reported an average of 3.4 characters on screen in the repeated condition and 4.2 in the random condition. Since numerosity judgements themselves are prone to distortion (Cheatham \& White 1952; Philippi et al. 2008), we tested participants on static displays involving multiple instances of the same or different stimuli. However, participants did not perceive any difference in simple numerosity across 'same' or 'different' conditions (Pariyadath \& Eagleman 2008). Collectively, these results suggest that repetition contracts the duration of visual persistence in the same manner in which it contracts durations at longer time scales. A contraction in the visual persistence of repeated stimuli leads to less temporal overlap, and hence fewer items are perceived to be present at once. The differential proliferation effect generalizes across stimuli such as pictures of everyday objects and short nonsense words such as 'abg' and 'hqe' (figure $2 b$, 'pictures' and 'non-words'). Again, these results are consistent with a theoretical framework in which subjective duration parallels repetition suppression: the events that seem to have a shorter duration (and thus, less on-screen overlap) are presumably those with neural responses diminished by repeated presentation.

\section{UNIFYING OTHER OBSERVATIONS UNDER A SINGLE FRAMEWORK}

First, these observations offer a framework for understanding several other illusions in the literature. For example, in the stopped clock illusion, the second hand of a clock seems to be momentarily frozen upon first glance, and then begins to tick at its expected pace (Yarrow et al. 2001, 2004; Park et al. 2003; Yarrow \& Rothwell 2003). In this framework, the successive stimuli are shrunken in perceived duration compared to the first.

Second, Nakajima and his colleagues have described an effect they call 'time shrinking': when two short stimuli are presented serially, the second is underestimated in duration (Nakajima et al. 1992, 2004; ten Hoopen et al. 1995; Arao et al. 2000; Sasaki et al. 2002). Furthermore, when the interval between the two stimuli increases, the effect of shrinkage goes away (Wearden \& Ferrara 1993; Wearden et al. 2002; Kanai \& Watanabe 2006). We hypothesize that both findings reflect a single phenomenon: the repetition of a stimulus contracts its perceived duration, and just as in neural data ( $\mathrm{Li}$ et al. 1993), the suppression recovers with time.

Third, there has long been an enduring mystery about flicker fusion: the smallest interval required for two flashes to be perceived as separate (also known as the two-flash fusion threshold) is greater than the smallest interval needed between successive flashes in a train (the CFFT; Herrick 1974). How could it be that two events, widely separated, could be perceived as united, when multiple events, closer together, can be distinguished? We here point out that the phenomenon can be easily explained in the context of repetition suppression. In the two-flash case, the visual persistence of the first flash overlaps with the appearance of the second flash, making them non-dissociable (figure 3). But in the continuously repeated case, the visible persistence of the flash contracts due to repetition suppression - and a faster train of flashes can thus be perceived as a series of separate events (Herrick 1974).

Finally, our hypothesis ties in directly with a recent report of 'change-related persistence': when a moving 
object undergoes a sudden, brief change, say in size or brightness, it is momentarily perceived as two separate objects (Moore et al. 2007). Moore and her colleagues proposed that if an object is understood by the visual system as a single object in motion, its visual persistence is reduced. But when the object changes in some attribute, it is registered as a different object and two separate instances of the object are perceived simultaneously, presumably because of an expanded visual persistence. This finding matches perfectly with our own, although there may be an advantage to interpreting the result in terms of repetition suppression rather than objecthood. This is because the notion of an object is binary-something either is an object or is not. But we have found that duration can be modified by the degree of novelty. For example, randomizing both letters and the colour of the letters in the proliferation paradigm leads to a higher perceived numerosity than changing the letters alone (figure 4). In other words, something can be more or less different, rather than being an object or not.

We now turn to 10 more reports in the psychophysics literature that appear to be consistent with the framework that the amplitude of the neural response maps onto subjective duration (table 1). The assumption of our framework is that stimulus manipulations leading to increased neural responses also lead to duration dilations; in table 1 , we provide evidence for this connection where available, but the connection remains to be tested or confirmed for other entries.

For example, increasing the luminance of an object (either physically or perceptually with brightness enhancement illusions) increases its perceived duration (Brigner 1986; Sperandio et al. 2008). Conversely, reducing the visibility of a stimulus (as stimuli are during a saccade) leads to duration compressions (Terao et al. 2008). More generally, Xuan et al. (2007) demonstrated that duration is dilated by the magnitude of a stimulus. Whether they manipulated brightness, size or numerosity, higher magnitude stimuli were perceived to have a longer duration than equal-length stimuli of smaller magnitude (Xuan et al. 2007). Ono \& Kawahara (2007) further demonstrated that the duration distortion depends on the perceived size of the stimulus, indicating that later visual processing plays a role (Ono \& Kawahara 2007). These findings, at least to a first approximation, parallel the electrophysiology: stimuli that are brighter (Barlow et al. 1978; Tikhomirov 1983; Maunsell et al. 1999), larger (Murray et al. 2006) and with higher numerosity (Roitman et al. 2007), all lead to higher firing rates.

As another example from table 1, subjective duration increases with the temporal frequency of a flickering stimulus (Kanai et al. 2006), but with an interesting pattern: duration dilations saturate as they approach temporal frequencies of $8 \mathrm{~Hz}$. Intriguingly, a parallel phenomenon can be found in the BOLD response to flicker: an increase in flicker rate leads to increasing activation in striate cortex, and the trend saturates at $8 \mathrm{~Hz}$ (Kaufmann et al. 2000).

There are several temporal illusions for which the corresponding physiology remains to be directly investigated-these are listed at the end of table 1 . Mostly, these involve the dynamics between the markers that define an interval. For example, filled intervals (a light that appears, remains on and then disappears) are perceived as longer than empty intervals (two brief flashes defining an interval) of equal physical duration (Ihle \& Wilsoncroft 1983). More generally, it has been long noted that the apparent duration of a sequence is dilated with increasing pattern complexity (Roelofs \& Zeeman 1951; Schiffman \& Bobko 1974), and those observations were followed by proposals that the brain estimates time based on the number of 'events' that occur (Fraisse 1963; Poynter 1989; Brown 1995). Although it seems intuitive that the brain expends more energy to represent a higher density of events per unit time, the available physiology literature does not appear to address these predictions directly; therefore, the later entries in the table await experimental testing.

\section{OTHER EVIDENCE FOR LOW-LEVEL MECHANISMS IN DURATION PERCEPTION}

The dependency of our framework on basic measures of energy expenditure suggests that the perception of time may be related to very low-level properties of neurons, rather than a highly cognitive algorithm. This view is consistent with several recent demonstrations. For example, Johnston et al. (2006) found that adaptation to a flickering stimulus led to duration distortions of subsequent stimuli; since the effect was spatially localized, this suggested a source of timing in early visual areas (Johnston et al. 2006). Similarly, the role of temporal frequency in duration distortions may also point to early levels of visual processing (Kanai et al. 2006). Finally, reductions in stimulus visibility - which are related to the transient responses of neurons (Macknik \& Livingstone 1998)-led to compressed subjective durations (Terao et al. 2008). All these findings suggest that low-level neural signatures of neurons will play an important role in duration perception.

\section{NEURAL RESPONSES AND THE SPEED OF REACTION}

The size of a neural response appears to map not only to subjective duration, but also inversely to motor reaction times. For example, the increase in perceived duration with increasing size (Xuan et al. 2007) and luminance (Sperandio et al. 2008) correlates with a decrease in reaction time (Plainis \& Murray 2000; Ono \& Kawahara 2007; Sperandio et al. 2008). Similarly, moving, looming or flickering stimuli appear to last longer in duration than static or receding stimuli (Roelofs \& Zeeman 1951; Brown 1995; Kanai et al. 2006; van Wassenhove et al. 2008) and also lead to faster reaction times (Mashhour 1964; Smeets \& Brenner 1995; Brenner \& Smeets 2003; López-Moliner 2005). An auditory stimulus lasts subjectively longer than a visual stimulus of equal duration (Wearden et al. 1998) and triggers a shorter reaction time (Woodworth \& Schlosberg 1954). Familiar words evoke longer perceived durations (Witherspoon \& Allan 1985) and shorter reaction times (Balota \& Spieler 1999) than unfamiliar ones. Luminance-defined stimuli appear longer in duration 


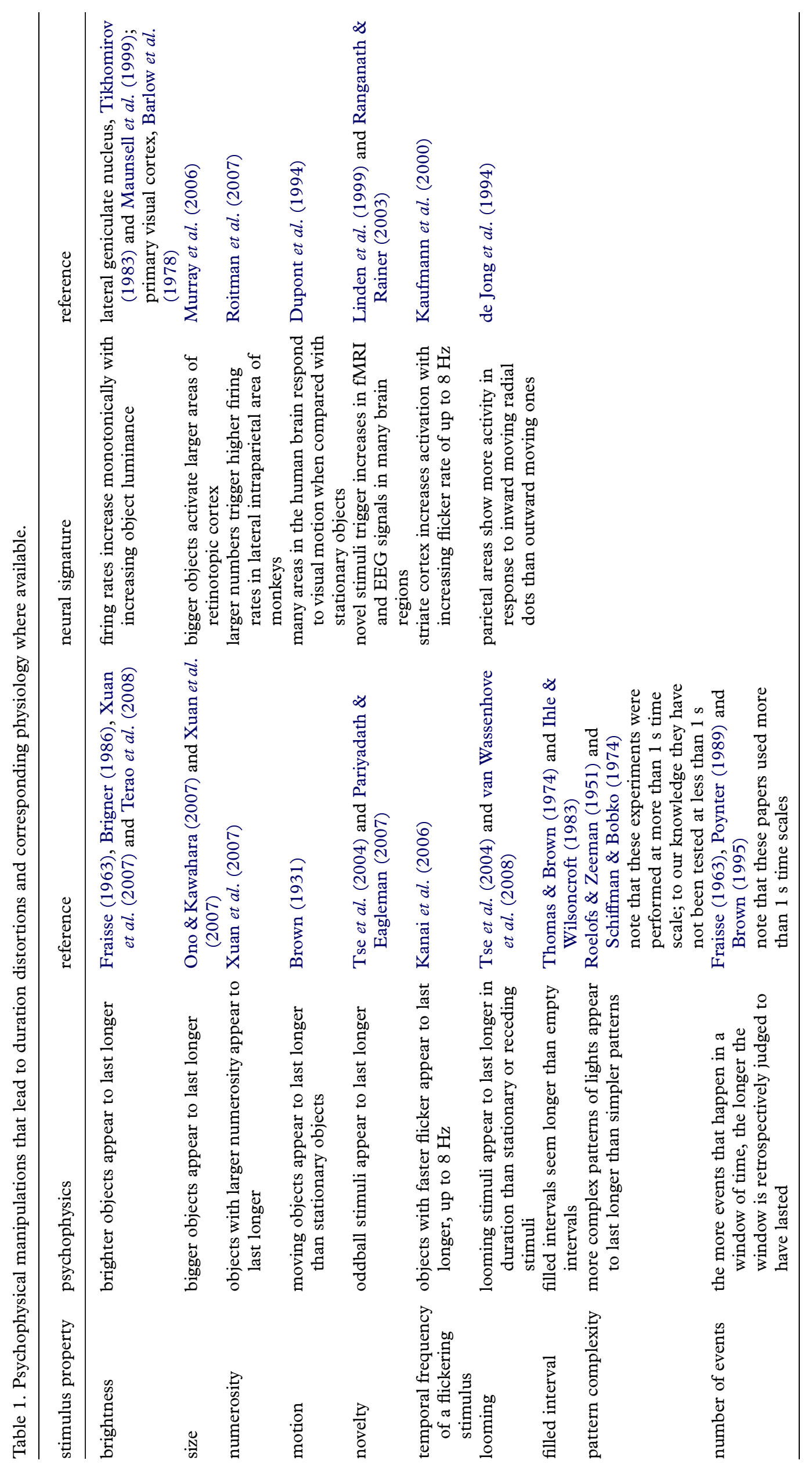


(a) two flashes

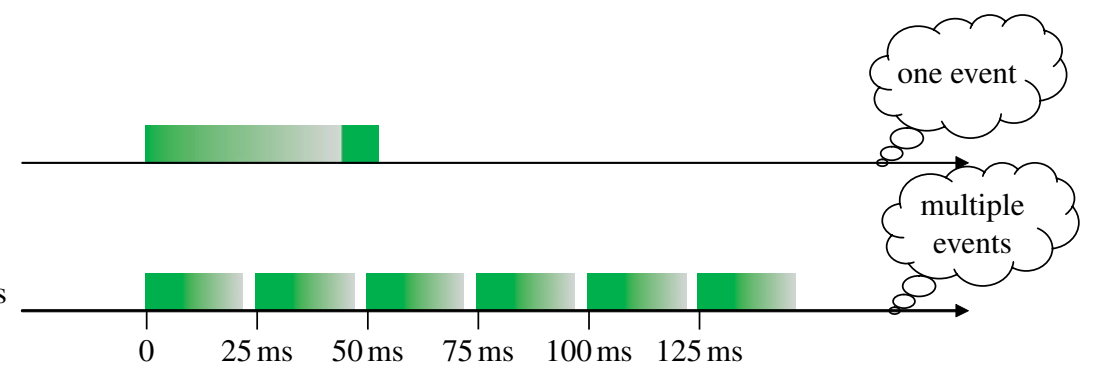

Figure 3. Explaining a mystery about flicker fusion. (a) The visual persistence of the first flash leads to the illusion of two flashes being one single flash. (b) A contraction in the visual persistence of flashes from repetition causes a train of flashes to be perceived as multiple events.

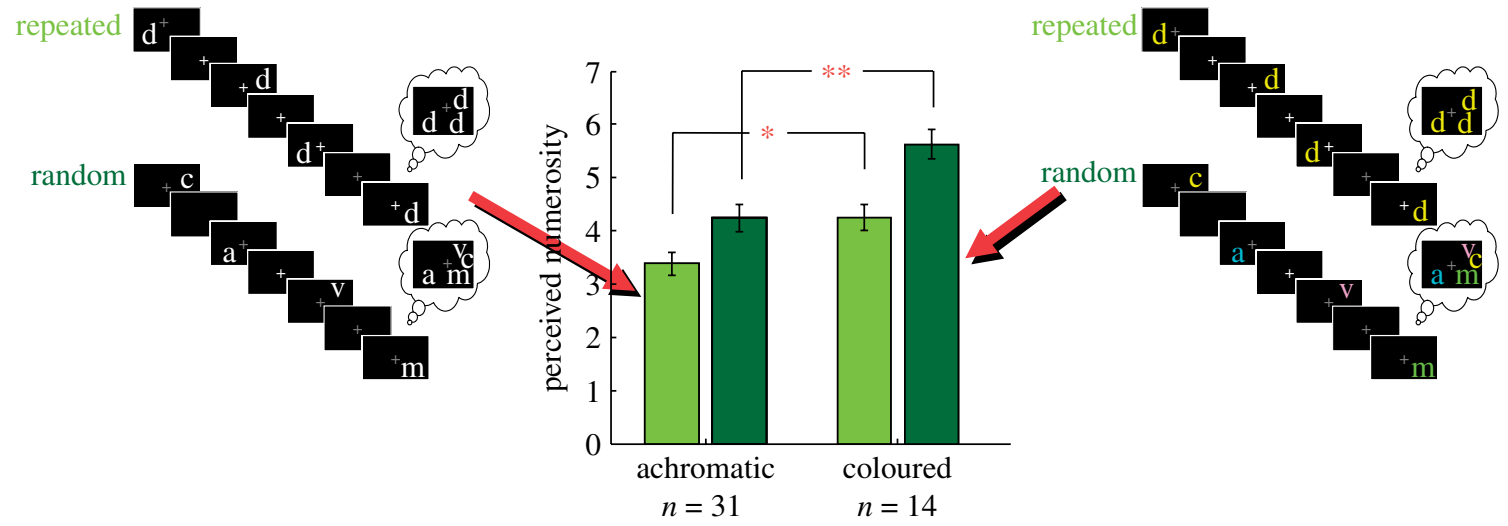

Figure 4. Adding a new dimension of novelty (in this case, colour) increases the overall perceived numerosity. Participants were tested on the proliferation paradigm using letters of the alphabet presented either in white ('achromatic') or in colours ('chromatic'). Within the chromatic condition, letters were either presented in the same colour or in different, but isoluminant, colours. We found that participants tend to report seeing more stimuli overall when a new stimulus attribute or a new degree of novelty is added to the stimulus $\left({ }^{*} p<0.05,{ }^{* *} p<0.01\right.$, paired $t$-test). Presumably, this is because the visual persistence of the stimuli is modulated by the degree of difference or similarity from the previously seen stimuli.

than equiluminant stimuli (Cicchini \& Tomassini 2008) and also lead to faster reaction times (McKeefry et al. 2003). With ageing, subjective durations decrease (Lustig 2003) as reaction times get longer (Jevas \& Yan 2001; Luchies et al. 2002; Rose et al. 2002; Der \& Deary 2006).

The idea that ties these observations together is that increased neural activity both allows the signal to move through the system more rapidly (faster reaction time) and translates into a longer subjective duration.

\section{MAGNITUDE}

Our hypothesis that perceived duration is influenced by the amount of neural activity may be related to magnitude considerations more generally. This is suggested by the fact that several illusions of duration have parallels in other forms of magnitude. For example, filled intervals are perceived as lasting longer than empty intervals (Thomas \& Brown 1974; Ihle \& Wilsoncroft 1983). A parallel to this illusion is found in the filled-extent or the Oppel-Kundt illusion: filled areas or volumes appear to be larger than empty ones (Bulatov et al. 1997). The 'tau' and 'kappa' effects provide additional evidence that magnitude in space and time interact with each other (Sarrazin et al. 2004): when three light flashes are presented serially to indicate two different intervals (of distance and duration), a longer temporal interval between two flashes leads to the interval being perceived as larger in distance (tau). Similarly, a larger spatial interval tends to be overestimated in duration (kappa).

Moreover, duration judgements are compressed during saccades (Eagleman 2005; Morrone et al. 2005): when subjects were asked to judge an interval between two flashes near in time to a saccade (by comparison with two more targets well after the saccade), durations were underestimated by about a factor of 2. Interestingly, the range from which the compression is observed-both before and after a saccade - is roughly the same range in which spatial compression is found (Honda 1991; Ross et al. 1997), suggesting for future research a possible common mechanism for time and space distortions.

\section{THE FRAMEWORK IS INCOMPLETE}

We have suggested that subjective duration reflects the size of neural response to a stimulus, but this hypothesis, if correct, will require a great deal of refinement. We cannot currently determine which neural activity will be critical: a particular window of time within a spike train; inhibitory versus excitatory firing; the involvement of particular cell types; and is post-synaptic firing more important than pre-synaptic release? Also, it is presently difficult to elaborate which brain regions will play critical roles. One manner in which this question can be answered is by focusing on repetition suppression (Grill-Spector et al. 2006). While many parts of the brain show a diminishment of neural response with repetition, all of them need not 
(a)

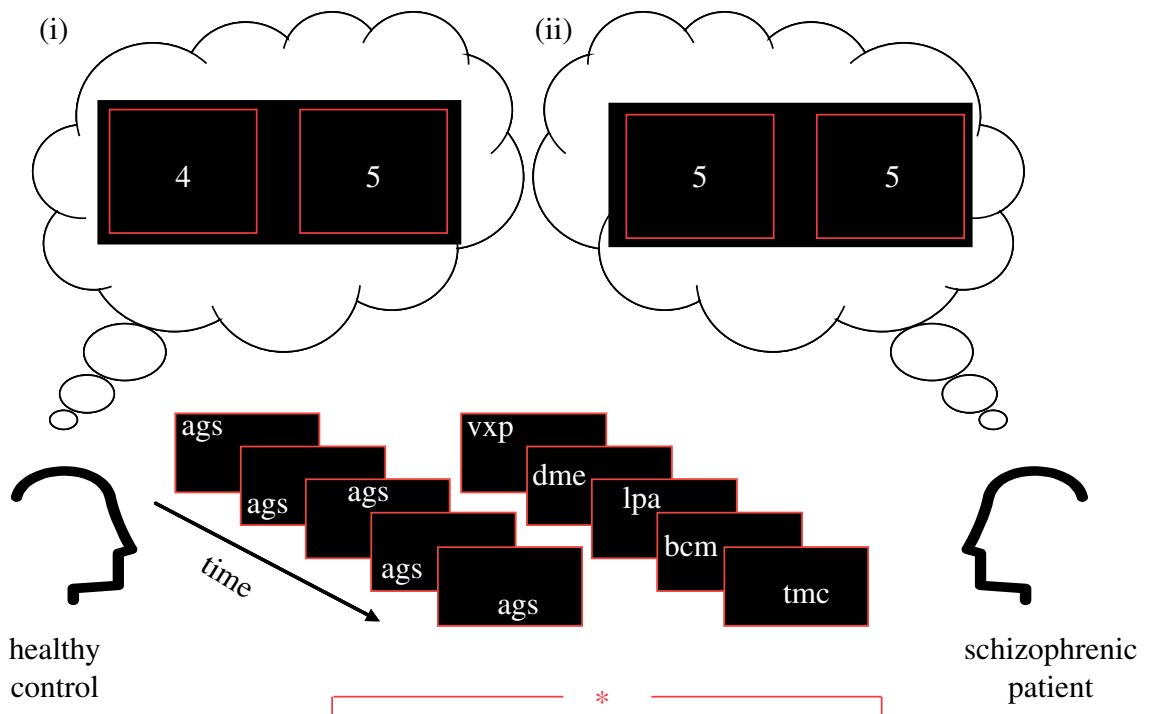

(b)

control

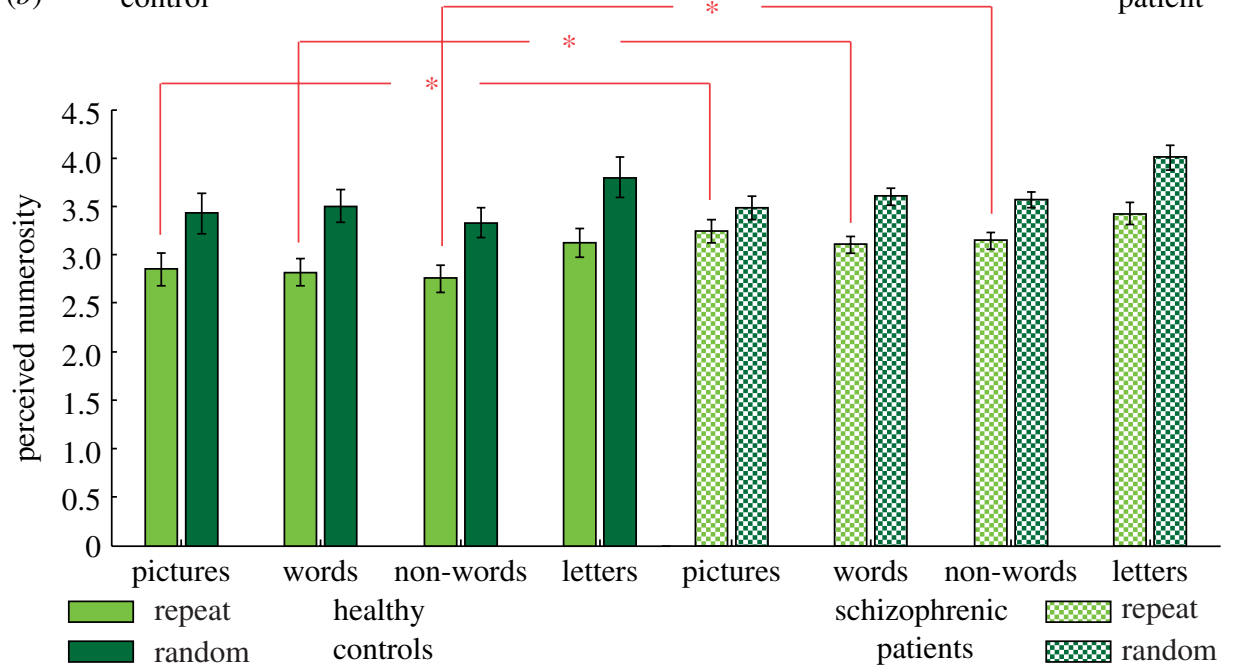

Figure 5. Proposed screening tool for schizophrenia. (a) Because schizophrenic patients show less repetition suppression to many types of stimuli, we hypothesized that they should report seeing more stimuli in the 'repeat' condition than healthy controls. (b) While schizophrenic patients report similar numerosities in the 'random' condition compared with healthy controls, they tend to report seeing significantly more stimuli simultaneously present in the repeat condition $\left({ }^{*} p<0.05\right.$, unpaired $t$-test $)$. By comparing the perceived numerosity for these two types of stimuli, an early screening might be made for schizophrenia.

contribute equally to subjective duration. By varying the type of stimulus or the stimulus characteristic that is being repeated, and by concomitantly examining the effect of repetition on its perceived duration, we may be able to tease out the role of various brain areas in the proposed mapping of duration and neural activity.

\section{SCHIZOPHRENIA}

Finally, our framework for duration has direct implications for understanding timing characteristics in schizophrenia. An impaired novelty response is a hallmark characteristic of schizophrenia, as evidenced by an impaired pre-pulse inhibition of the startle response (Hong et al. 2007), impaired mismatch negativity (Javitt et al. 1998; Light \& Braff 2005) and poor oddball detection (Kiehl \& Liddle 2001). Relatedly, schizophrenics show a lowered CFFT (Black et al. 1975), and a lower sensitivity for detecting flicker (Slaghuis \& Bishop 2001). These findings are consistent with electrophysiological measures that show a reduced or absent repetition suppression in schizophrenics, presumably because of a deficit in cortical inhibition (Daskalakis et al. 2002). Roughly speaking, to a schizophrenic brain everything appears novel, and repetition has little efficacy in reducing neural responses.

Given the evidence of impaired repetition suppression in schizophrenia, we hypothesized that schizophrenic patients should perceive a smaller contraction in visual persistence with repetition when compared with controls (figure 5). To test this, we used the proliferation effect (figure 2) and found that while schizophrenic patients perceive similar numerosities on the random condition to age- and IQ-matched healthy controls, their repeated numerosities were significantly higher (Gandhi et al. 2007). This trend was observed with stimuli including letters, words, non-words and pictures-and suggests that the proliferation effect could be potentially used as a screening tool for schizophrenia.

We are currently exploring implications of our framework for other disorders. For example, in autism, a more specific impairment to repetition suppression is seen. Autistic patients show a diminished repetition suppression response to familiar faces even as their response is preserved for inanimate objects (Dawson et al. 2005). This makes a testable prediction about the 
perceived duration of repeated objects and faces in autism: while the subjective duration of repeated objects will contract, that of familiar faces will not.

\section{CONCLUSIONS}

We have presented evidence that supports a new understanding of short time-scale subjective duration: the duration assigned to a stimulus reflects the magnitude of the neural response to the stimulus (Pariyadath \& Eagleman 2007; Eagleman 2008). We suggest the speculative possibility that subjective duration might be considered a qualia, a property that is assigned to stimuli in the same way that colour is bound to objects, or motion can be 'painted on' to stimuli by the visual system (Crick \& Koch 2003). In other words, duration may be an attribute that is painted on to interpretations of events in the world.

The framework presented here might appear closely related to an attentional hypothesis, i.e. the suggestion that attended objects will appear to last longer (Rose \& Summers 1995; Tse et al. 2004). But note that while the two hypotheses are similar, they are not identical. For example, the attentional hypothesis should predict that increasing the emotional salience of the oddball stimulus (e.g. a tarantula instead of a shoe) should result in increased duration dilation. However, no such increase occurs (Pariyadath \& Eagleman 2007), suggesting that the unpredictability of the oddball (and hence the release from suppression) is the key ingredient. Moreover, we favour a neural amplitude formulation because it is directly falsifiable with measures of neural activity, while the concept of attention has traditionally proven more difficult to pin down.

We are currently working on ways to highlight and test this distinction further. For example, a point of divergence between the attentional and neural amplitude hypotheses may be discoverable by manipulating mental load. Repetition suppression is not modulated by changes in processing load ( $\mathrm{Xu}$ et al. 2007). Therefore, if the perceived duration of a repeated stimulus is a function of attentional resources, it will be influenced by manipulations of processing load; if it is a function of neural amplitude, processing load will not have an influence. Experiments such as these should allow us to judge the relative merits of the attentional and neural amplitude frameworks.

Moreover, while it has been previously proposed that increases in attention are responsible for increases in duration (Rose \& Summers 1995; Tse et al. 2004), we would like to suggest two variations on this hypothesis. First, our formulation suggests that a larger neural response (presumably reflecting less efficient encoding) is the basis for both the increased attention and the larger duration. Second, and more speculatively, it could be that increases in perceived duration drive attention, essentially allowing more perceptual opportunity for the system to 'grab onto' a stimulus. In other words, because the common correlation between duration and attention does not give a causal arrow, it is logically possible that they are driven by a common driver, or that the arrow points in the unexpected direction from duration to attention rather than the other way around.
We have recently become aware that Marchetti (2008) has proposed an idea similar to ours, writing that the sensation of time comes from perceiving how much 'effort' is made 'by the organ of attention'. What he calls effort by the organ of attention is what we call energy expenditure in neural networks. (Although they are similar concepts, ours may be slightly more amenable to measurement.) Marchetti further notes that 'the capacity to directly perceive the effort made by our organs, in general, is an innate one: it is precisely this capacity that gives us the possibility of feeling the fatigue of our various organs, and of having sensations of exhaustion, weariness, tiredness, freshness, etc'. Marchetti (2008) goes on to suggest that only effort devoted to the temporal aspects of a scene will influence duration; we currently remain agnostic about which aspects of neural activity will be implicated, but we will use his suggestion as one possible framework for guiding experiments and measurements. (Finally, we note that although Marchetti calls his hypothesis a revision of one from Ernst Mach, it should be noted that Mach's original proposal addressed temporal order, not duration. Specifically, he proposed that earlier and later could be tagged by the state of the monotonically increasing fatigue of the 'organ of attention', Mach 1890).

We have restricted our discussion to events in the time scale of tens to hundreds of milliseconds as currently there is not enough evidence to indicate that similar mechanisms come into play in duration judgements at longer time scales. But certain principles that we observe at brief time scales appear to hold at longer ones. For example, there are many anecdotal reports of the return leg of a journey being shorter in duration than the onward leg, possibly because of the novelty of scenery wearing off as one travels back along the same route.

If our hypothesis relating neural energy expenditure to subjective duration proves to be on the correct track, it will almost certainly be refined beyond recognition in the future: as noted above, it may turn out that only certain cell types are involved, and/or only a specific part of the spike train that is important; it may even be that intracellular dynamics are the key players, not the spikes. Whatever be the refinements in the future, we hope this hypothesis may provide a good starting point for a new framework and new experiments that can put it to the test.

We thank the two anonymous reviewers for their helpful comments on an earlier draft of this manuscript. Funding for the research was provided by National Institutes of Health grant RO1 NS053960 (D.M.E.).

\section{REFERENCES}

Arao, H., Suetomi, D. \& Nakajima, Y. 2000 Does timeshrinking take place in visual temporal patterns? Perception 29, 819-830. (doi:10.1068/p2853)

Balota, D. A. \& Spieler, D. H. 1999 Word frequency, repetition, and lexicality effects in word recognition tasks: beyond measures of central tendency. F. Exp. Psychol. Gen. 128, 32-55. (doi:10.1037/0096-3445.128.1.32)

Barlow, R. B., Snodderly, D. M. \& Swadlow, H. A. 1978 Intensity coding in primate visual system. Exp. Brain Res. 31, 163-177. (doi:10.1007/BF00237597) 
Black, S., Franklin, L. M., de Silva, F. P. \& Wijewickrama, H. S. 1975 The flicker-fusion threshold in schizophrenia and depression. N. Z. Med. F. 81, 244-246.

Bowen, R. W., Pola, J. \& Matin, L. 1974 Visual persistence: effects of flash luminance, duration and energy. Vision Res. 14, 295-303. (10.1016/0042-6989(74)90079-0)

Brenner, E. \& Smeets, J. B. 2003 Perceptual requirements for fast manual responses. Exp. Brain Res. 153, 246-252. (doi:10.1007/s00221-003-1598-y)

Brigner, W. L. 1986 Effect of perceived brightness on perceived time. Percept. Mot. Skills 63, 427-430.

Brown, J. F. 1931 Motion expands perceived time. On time perception in visual movement fields. Psychol. Forsch. 14, 233-248. (doi:10.1007/BF00403874)

Brown, S. W. 1995 Time, change, and motion: the effects of stimulus movement on temporal perception. Percept. Psychophys. 57, 105-116.

Buckner, R. L., Petersen, S. E., Ojemann, J. G., Miezin, F. M., Squire, L. R. \& Raichle, M. E. 1995 Functional anatomical studies of explicit and implicit memory retrieval tasks. 7. Neurosci. 15, 12-29.

Buhusi, C. V. \& Meck, W. H. 2005 What makes us tick? Functional and neural mechanisms of interval timing. Nat. Rev. Neurosci. 6, 755-765. (doi:10.1038/nrn1764)

Bulatov, A., Bertulis, A. \& Mickiene, L. 1997 Geometrical illusions: study and modelling. Biol. Cybern. 77, 395-406. (doi:10.1007/s004220050399)

Buonomano, D. V. \& Mauk, M. D. 1994 Neural codes and distributed representations: foundations of neural computation. Neural Comput. 6, 38-55. (doi:10.1162/neco.1994. 6.1.38)

Buonomano, D. V. \& Merzenich, M. M. 1995 Temporal information transformed into a spatial code by a neural network with realistic properties. Science 267, 1028-1030. (doi:10.1126/science.7863330)

Cheatham, P. G. \& White, C. T. 1952 Temporal numerosity: I. Perceived number as a function of flash number and rate. F. Exp. Psychol. 44, 447-451. (doi:10.1037/ h0061192)

Cicchini, G. M. \& Tomassini, A. 2008 Perception of duration in the parvocellular system. Perception 37, 69.

Creelman, C. D. 1962 Human discrimination of auditory duration. F. Acoust. Soc. Am. 34, 528-593. (doi:10.1121/ $1.1918172)$

Crick, F. H. C. C. \& Koch, C. 2003 A framework for consciousness. Nat. Neurosci. 6, 119-126. (doi:10.1038/ nn0203-119)

Daskalakis, Z. J., Christensen, B. K., Chen, R., Fitzgerald, P. B., Zipursky, R. B. \& Kapur, S. 2002 Evidence for impaired cortical inhibition in schizophrenia using transcranial magnetic stimulation. Arch. Gen. Psychiatry 59, 347-354. (doi:10.1001/archpsyc.59.4.347)

Dawson, G., Webb, S. J. \& McPartland, J. 2005 Understanding the nature of face processing impairment in autism: insights from behavioral and electrophysiological studies. Dev. Neuropsychol. 27, 403-424. (doi:10.1207/ s15326942dn2703_6)

de Jong, B. M., Shipp, S., Skidmore, B., Frackowiak, R. S. \& Zeki, S. 1994 The cerebral activity related to the visual perception of forward motion in depth. Brain 117(Pt 5), 1039-1054. (doi:10.1093/brain/117.5.1039)

Der, G. \& Deary, I. J. 2006 Age and sex differences in reaction time in adulthood: results from the United Kingdom health and lifestyle survey. Psychol. Aging 21, 62-73. (doi:10.1037/0882-7974.21.1.62)

Desimone, R. \& Duncan, J. 1995 Neural mechanisms of selective visual attention. Annu. Rev. Neurosci. 18, 193-222. (doi:10.1146/annurev.ne.18.030195.001205)

Di Lollo, V. 1977 Temporal characteristics of iconic memory. Nature 267, 241-243. (doi:10.1038/267241a0)
Dupont, P., Orban, G. A., De Bruyn, B., Verbruggen, A. \& Mortelmans, L. 1994 Many areas in the human brain respond to visual motion. F. Neurophysiol. 72, 1420-1424.

Eagleman, D. M. 2005 Distortions of time during rapid eye movements. Nat. Neurosci. 8, 850-851.

Eagleman, D. M. 2008 Human time perception and its illusions. Curr. Opin. Neurobiol. 18, 131-136. (doi:10. 1016/j.conb.2008.06.002)

Eagleman, D. M., Tse, P. U., Buonomano, D., Janssen, P., Nobre, A. C. \& Holcombe, A. O. 2005 Time and the brain: how subjective time relates to neural time. F. Neurosci. 25, 10 369-10 371. (doi:10.1523/JNEUROSCI.3487-05.2005)

Efron, R. 1970 The minimum duration of a perception. Neurophysiologia 8, 57-63. (doi:10.1016/0028-3932(70) 90025-4)

Fahy, F. L., Riches, I. P. \& Brown, M. W. 1993 Neuronal activity related to visual recognition memory: long-term memory and the encoding of recency and familiarity information in the primate anterior and medial inferior temporal and rhinal cortex. Exp. Brain Res. 96, 457-472. (doi:10.1007/BF00234113)

Fraisse, P. 1963 The psychology of time. New York, NY: Harper and Row.

Gandhi, S. K., Pariyadath, V., Wassef, A. A. \& Eagleman, D. M. 2007 Timing judgments in schizophrenia. Society for Neuroscience abstract, San Diego, CA. 806.13.

Grill-Spector, K., Henson, R. \& Martin, A. 2006 Repetition and the brain: neural models of stimulus-specific effects. Trends Cogn. Sci. 10, 14-23. (doi:10.1016/j.tics.2005.11. 006)

Henson, R. N. A. \& Rugg, M. 2001 Effects of stimulus repetition on latency of the BOLD impulse response. NeuroImage 13， 683. (doi:10.1016/S1053-8119(01) 92026-9)

Henson, R. N. A. \& Rugg, M. D. 2003 Neural response suppression, haemodynamic repetition effects, and behavioural priming. Neuropsychologia 41, 263-270. (doi:10. 1016/S0028-3932(02)00159-8)

Herrick, R. M. 1974 Frequency thresholds for two-flash flicker and critical flicker: why they differ. Percept. Psychophys. 15, 79-82.

Hodinott-Hill, I., Thilo, K. V., Cowey, A. \& Walsh, V. 2002 Auditory chronostasis: hanging on the telephone. Curr. Biol. 12, 1779-1781. (doi:10.1016/S0960-9822(02)01219-8)

Honda, H. 1991 The time courses of visual mislocalization and of extraretinal eye position signals at the time of vertical saccades. Vision Res. 31, 1915-1921. (doi:10. 1016/0042-6989(91)90186-9)

Hong, L. E., Summerfelt, A., Wonodi, I., Adami, H., Buchanan, R. W. \& Thaker, G. K. 2007 Independent domains of inhibitory gating in schizophrenia and the effect of stimulus interval. Am. F. Psychiatry 164, 61-65. (doi:10.1176/appi.ajp.164.1.61)

Ihle, R. C. \& Wilsoncroft, W. E. 1983 The filled-duration illusion: limits of duration of interval and auditory fillers. Percept. Mot. Skills 56, 655-660.

Ishai, A., Bikle, P. C. \& Ungerleider, L. G. 2006 Temporal dynamics of face repetition suppression. Brain Res. Bull. 70, 289-295. (doi:10.1016/j.brainresbull.2006.06.002)

Ivry, R. B. \& Schlerf, J. E. 2008 Dedicated and intrinsic models of time perception. Trends Cogn. Sci. 12, 273-280. (doi:10.1016/j.tics.2008.04.002)

Javitt, D. C., Grochowski, S., Shelley, A. \& Ritter, W. 1998 Impaired mismatch negativity (MMN) generation in schizophrenia as a function of stimulus deviance, probability, and interstimulus/interdeviant interval. Electroencephalogr. Clin. Neurophysiol. 108, 143-153. (doi:10.1016/ S0168-5597(97)00073-7) 
Jevas, S. \& Yan, J. H. 2001 The effect of aging on cognitive function: a preliminary quantitative review. Res. Q. Exerc. Sport 72, A-49.

Johnston, A., Arnold, D. H. \& Nishida, S. 2006 Spatially localized distortions of event time. Curr. Biol. 16, 472-479. (doi:10.1016/j.cub.2006.01.032)

Kanai, R. \& Watanabe, M. 2006 Visual onset expands subjective time. Percept. Psychophys. 68, 1113-1123.

Kanai, R., Paffen, C. L., Hogendoorn, H. \& Verstraten, F. A. 2006 Time dilation in dynamic visual display. F. Vis. 6, 1421-1430. (doi:10.1167/6.12.8)

Karmarkar, U. R. \& Buonomano, D. V. 2007 Timing in the absence of clocks: encoding time in neural network states. Neuron 53, 427-438. (doi:10.1016/j.neuron.2007.01.006)

Kaufmann, C., Elbel, G. K., Gössl, C., Putz, B. \& Auer, D. P. 2000 Gender differences in a graded visual stimulation paradigm for fMRI are limited to striate visual cortex. In Proc. Int. Soc. Mag. Reson. Med. 8, 203.

Kiehl, K. A. \& Liddle, P. F. 2001 An event-related functional magnetic resonance imaging study of an auditory oddball task in schizophrenia. Schizophr. Res. 48, 159-171. (doi:10.1016/S0920-9964(00)00117-1)

Lewis, P. A. \& Miall, R. C. 2003 Distinct systems for automatic and cognitively controlled time measurement: evidence from neuroimaging. Curr. Opin. Neurobiol. 13, 250-255. (doi:10.1016/S0959-4388(03)00036-9)

Li, L., Miller, E. K. \& Desimone, R. 1993 The representation of stimulus familiarity in anterior inferior temporal cortex. F. Neurophysiol. 69, 1918-1929.

Light, G. A. \& Braff, D. L. 2005 Mismatch negativity deficits are associated with poor functioning in schizophrenia patients. Arch. Gen. Psychiatry 62, 127-136. (doi:10.1001/ archpsyc.62.2.127)

Lin, J. M., Bohland, J. W., Andrews, P., Burns, G. A., Allen, C. B. \& Mitra, P. P. 2008 An analysis of the abstracts presented at the annual meetings of the Society for Neuroscience from 2001 to 2006. PLoS ONE 3, e2052. (doi:10.1371/journal.pone.0002052)

Linden, D. E., Prvulovic, D., Formisano, E., Vollinger, M., Zanella, F. E., Goebel, R. \& Dierks, T. 1999 The functional neuroanatomy of target detection: an fMRI study of visual and auditory oddball tasks. Cereb. Cortex 9, 815-823. (doi:10.1093/cercor/9.8.815)

López-Moliner, J. 2005 Reaction time asymmetries between expansion and contraction. Psicológica 26, 139-146.

Luchies, C. W., Schiffman, J., Richards, L. G., Thompson, M. R., Bazuin, D. \& DeYoung, A. J. 2002 Effects of age, step direction, and reaction condition on the ability to step quickly. F. Gerontol. A Biol. Sci. Med. Sci. 57, M246-M249.

Lustig, C. 2003 Grandfather's clock: attention and interval timing in older adults. In Functional and neural mechanisms of interval timing (ed. W. H. Meck), pp. 261-293. Boca Raton, FL: CRC Press.

Mach, E. 1890 Contributions to the analysis of the sensations. La Salle, IL: The Open Court Publishing Company.

Macknik, S. L. \& Livingstone, M. S. 1998 Neuronal correlates of visibility and invisibility in the primate visual system. Nat. Neurosci. 1, 144-149. (doi:10.1038/393)

Marchetti, G. 2008 Studies on time: a proposal on how to get out of circularity. Cogn. Process. 10, 7-40. (doi:10.1007/ s10339-008-0215-1)

Mashhour, M. 1964 Psychophysical relations in the perception of velocity. Stockholm, Sweden: Almquist.

Mauk, M. D. \& Buonomano, D. V. 2004 The neural basis of temporal processing. Annu. Rev. Neurosci. 27, 307-340. (doi:10.1146/annurev.neuro.27.070203.144247)

Maunsell, J. H., Ghose, G. M., Assad, J. A., McAdams, C. J., Boudreau, C. E. \& Noerager, B. D. 1999 Visual response latencies of magnocellular and parvocellular LGN neurons in macaque monkeys. Vis. Neurosci. 16, 1-14. (doi:10.1017/S0952523899156177)

McKeefry, D. J., Parry, N. R. \& Murray, I. J. 2003 Simple reaction times in color space: the influence of chromaticity, contrast, and cone opponency. Invest. Ophthalmol. Vis. Sci. 44, 2267-2276. (doi:10.1167/iovs.02-0772)

Moore, C. M., Mordkoff, J. T. \& Enns, J. T. 2007 The path of least persistence: object status mediates visual updating. Vision Res. 47, 1624-1630. (doi:10.1016/ j.visres.2007.01.030)

Morrone, M. C., Ross, J. \& Burr, D. 2005 Saccadic eye movements cause compression of time as well as space. Nat. Neurosci. 8, 950-954. (doi:10.1038/nn1488)

Murray, S. O., Boyaci, H. \& Kersten, D. 2006 The representation of perceived angular size in human primary visual cortex. Nat. Neurosci. 9, 429-434. (doi:10.1038/ nn1641)

Nakajima, Y., ten Hoopen, G., Hilkhuysen, G. \& Sasaki, T. 1992 Time-shrinking: a discontinuity in the perception of auditory temporal patterns. Percept. Psychophys. 51, 504-507.

Nakajima, Y., ten Hoopen, G., Sasaki, T., Yamamoto, K., Kadota, M., Simons, M. \& Suetomi, D. 2004 Timeshrinking: the process of unilateral temporal assimilation. Perception 33, 1061-1079. (doi:10.1068/p5061)

Noguchi, Y., Inui, K. \& Kakigi, R. 2004 Temporal dynamics of neural adaptation effect in the human visual ventral stream. F. Neurosci. 24, 6283-6290. (doi:10.1523/ JNEUROSCI.0655-04.2004)

Ono, F. \& Kawahara, J. 2007 The subjective size of visual stimuli affects the perceived duration of their presentation. Percept. Psychophys. 69, 952-957.

Pariyadath, V. \& Eagleman, D. 2007 The effect of predictability on subjective duration. PLOS ONE 2, e1264. (doi:10.1371/journal.pone.0001264)

Pariyadath, V. \& Eagleman, D. M. 2008 Brief subjective durations contract with repetition. F. Vis. 8, 1-6. (doi:10. $1167 / 8.16 .11)$

Park, J., Schlag-Rey, M. \& Schlag, J. 2003 Voluntary action expands perceived duration of its sensory consequence. Exp. Brain Res. 149, 527-529. (doi:10.1007/s00221-0031376-x)

Philippi, T. G., van Erp, J. B. \& Werkhoven, P. J. 2008 Multisensory temporal numerosity judgment. Brain Res. 1242, 116-125. (doi:10.1016/j.brainres.2008.05.056)

Plainis, S. \& Murray, I. J. 2000 Neurophysiological interpretation of human visual reaction times: effect of contrast, spatial frequency and luminance. Neuropsychologia 38, 1555-1564. (doi:10.1016/S0028-3932 (00)00100-7)

Poynter, W. D. 1989 Judging the duration of time intervals: a process of remembering segments of experience. In Time and human cognition: a life-span perspective (ed. I. L. D. Zakay), pp. 305-321. Amsterdam, The Netherlands: Elsevier.

Rainer, G. \& Miller, E. K. 2000 Effects of visual experience on the representation of objects in the prefrontal cortex. Neuron 27, 179-189. (doi:10.1016/S0896-6273(00)00019-2)

Rammsayer, T. H. 1999 Neuropharmacological evidence for different timing mechanisms in humans. Q. F. Exp. Psychol. B 52, 273-286. (doi:10.1080/027249899391070)

Ranganath, C. \& Rainer, G. 2003 Neural mechanisms for detecting and remembering novel events. Nat. Rev. Neurosci. 4, 193-204. (doi:10.1038/nrn1052)

Roelofs, C. O. Z. \& Zeeman, W. P. C. 1951 Influence of different sequences of optical stimuli on the estimation of duration of a given interval of time. Acta Psychol. 8, 89-128. (doi:10.1016/0001-6918(51)90007-8) 
Roitman, J. D., Brannon, E. M. \& Platt, M. L. 2007 Monotonic coding of numerosity in macaque lateral intraparietal area. PLoS Biol. 5, e208. (doi:10.1371/ journal.pbio.0050208)

Rose, D. \& Summers, J. 1995 Duration illusions in a train of visual stimuli. Perception 24, 1177-1187. (doi:10.1068/ p241177)

Rose, S. A., Feldman, J. F., Jankowski, J. J. \& Caro, D. M. 2002 A longitudinal study of visual expectation and reaction time in the first year of life. Child Dev. 73, 47-61. (doi:10.1111/1467-8624.00391)

Ross, J., Morrone, M. C. \& Burr, D. C. 1997 Compression of visual space before saccades. Nature 386, 598-601. (doi:10.1038/386598a0)

Sarrazin, J. C., Giraudo, M. D., Pailhous, J., Bootsma, R. J. \& Giraudo, M. D. 2004 Dynamics of balancing space and time in memory: tau and kappa effects revisited. F. Exp. Psychol. Hum. Percept. Perform. 30, 411-430. (doi:10. 1037/0096-1523.30.3.411)

Sasaki, T., Suetomi, D., Nakajima, Y. \& ten Hoopen, G. 2002 Time-shrinking, its propagation, and Gestalt principles. Percept. Psychophys. 64, 919-931.

Schiffman, H. R. \& Bobko, D. J. 1974 Effects of stimulus complexity on the perception of brief temporal intervals. F. Exp. Psychol. 103, 156-159. (doi:10.1037/h0036794)

Slaghuis, W. L. \& Bishop, A. M. 2001 Luminance flicker sensitivity in positive- and negative-symptom schizophrenia. Exp. Brain Res. 138, 88-99. (doi:10.1007/ s002210100683)

Smeets, J. B. \& Brenner, E. 1995 Perception and action are based on the same visual information: distinction between position and velocity. F. Exp. Psychol. Hum. Percept. Perform. 21, 19-31. (doi:10.1037/0096-1523.21.1.19)

Sperandio, I., Savazzi, S., Marzi, C. A. \& Gregory, R. 2008 Does reaction time depend upon perceived or retinal stimulus size? Perception 37, 14.

ten Hoopen, G., Hartsuiker, R., Sasaki, T., Nakajima, Y., Tanaka, M. \& Tsumura, T. 1995 Auditory isochrony: time shrinking and temporal patterns. Perception 24, 577-593. (doi:10.1068/p240577)

Terao, M., Watanabe, J., Yagi, A. \& Nishida, S. 2008 Reduction of stimulus visibility compresses apparent time intervals. Nat. Neurosci. 11, 541-542. (doi:10.1038/ nn.2111)

Thomas, E. A. C. \& Brown, I. 1974 Time perception and the filled-duration illusion. Percept. Psychophys. 16, 449-458.

Tikhomirov, A. S. 1983 Characteristics of the coding of light stimulus intensity by visual cortex neurons during light adaptation in the cat. Neirofiziologiia 15, 211-217.

Treisman, M. 1963 Temporal discrimination and the indifference interval: implications for a model of the 'internal clock'. Psychol. Monogr. 77, 1-31.
Tse, P. U., Intriligator, J., Rivest, J. \& Cavanagh, P. 2004 Attention and the subjective expansion of time. Percept. Psychophys. 66, 1171-1189.

Ulrich, R., Nitschke, J. \& Rammsayer, T. 2006 Perceived duration of expected and unexpected stimuli. Psychol. Res. 70, 77-87. (doi:10.1007/s00426-004-0195-4)

van Wassenhove, V., Buonomano, D. V., Shimojo, S. \& Shams, L. 2008 Distortions of subjective time perception within and across senses. PLOS ONE 3, e1437. (doi:10. 1371/journal.pone.0001437)

Wark, B., Lundstrom, B. N. \& Fairhall, A. 2007 Sensory adaptation. Curr. Opin. Neurobiol. 17, 423-429. (doi:10. 1016/j.conb.2007.07.001)

Wearden, J. H. \& Ferrara, A. 1993 Subjective shortening in humans' memory for stimulus duration. Q. F. Exp. Psychol. B 46, 163-186.

Wearden, J. H., Edwards, H., Fakhri, M. \& Percival, A. 1998 Why 'sounds are judged longer than lights': application of a model of the internal clock in humans. Q. F. Exp. Psychol. B 51, 97-120.

Wearden, J. H., Parry, A. \& Stamp, L. 2002 Is subjective shortening in human memory unique to time representations? Q. F. Exp. Psychol. B 55, 1-25. (doi:10.1080/ 02724990143000108)

Wiggs, C. L. \& Martin, A. 1998 Properties and mechanisms of perceptual priming. Curr. Opin. Neurobiol. 8, 227-233. (doi:10.1016/S0959-4388(98)80144-X)

Witherspoon, D. \& Allan, L. G. 1985 The effect of a prior presentation on temporal judgments in a perceptual identification task. Mem. Cogn. 13, 101-111.

Woodworth, R. S. \& Schlosberg, H. 1954 Experimental psychology. New York, NY: Henry Holt.

$\mathrm{Xu}$, Y., Turk-Browne, N. B. \& Chun, M. M. 2007 Dissociating task performance from fMRI repetition attenuation in ventral visual cortex. F. Neurosci. 27, 5981-5985. (doi:10.1523/JNEUROSCI.5527-06.2007)

Xuan, B., Zhang, D., He, S. \& Chen, X. 2007 Larger stimuli are judged to last longer. F. Vis. 7, 1-5. (doi:10. $1167 / 7.10 .2)$

Yamazaki, T. \& Tanaka, S. 2005 Neural modeling of an internal clock. Neural Comput. 17, 1032-1058. (doi:10. 1162/0899766053491850)

Yarrow, K. \& Rothwell, J. C. 2003 Manual chronostasis: tactile perception precedes physical contact. Curr. Biol. 13, 1134-1139. (doi:10.1016/S0960-9822(03) 00413-5)

Yarrow, K., Haggard, P., Heal, R., Brown, P. \& Rothwell, J. C. 2001 Illusory perceptions of space and time preserve cross-saccadic perceptual continuity. Nature 414, 302-305. (doi:10.1038/35104551)

Yarrow, K., Johnson, H., Haggard, P. \& Rothwell, J. C. 2004 Consistent chronostasis effects across saccade categories imply a subcortical efferent trigger. F. Cogn. Neurosci. 16, 839-847. (doi:10.1162/089892904970780) 